\title{
USO DE GEL DE LIDOCAÍNA INTRARECTAL EN LAS BIOPSIAS TRANSRECTALES ECODIRIGIDAS DE PRÓSTATA
}

\author{
J.M. GARCÍA MEDIERO*, L. MARTÍNEZ-PIÑEIRO LORENZO, C. NÚÑEZ MORA, \\ F. RAMÓN DE FATA CHILLÓN, J.L. CRUZ JIMENO, S. ALONSO Y GREGORIO, \\ J.J. DE LA PEÑA BARTHEL
}

\begin{abstract}
Servicio de Urología. *Hospital Universitario de Getafe. Hospital Universitario La Paz. Universidad Autónoma de Madrid. Madrid.
\end{abstract}

Actas Urol Esp. 27 (10): 793-796, 2003

\section{RESUMEN}

USO DE GEL DE LIDOCAÍNA INTRARECTAL EN LAS BIOPSIAS TRANSRECTALES ECODIRIGIDAS DE PRÓSTATA

OBJETIVO: Valoramos de una manera cuantitativa el grado de molestias y dolor de las biopsias y evaluamos la eficacia del gel de lidocaína intrarectal.

MÉTODO: Realizamos un total de 140 biopsias transrectales de próstata ecodirigidas (BTPE) por sospecha de adenocarcinoma prostático. Los pacientes fueron incluidos de forma aleatoria y homogénea en dos brazos, uno recibió gel de lidocaína, $20 \mathrm{mg}$ (Xylocaina ${ }^{\circledR}$ ), intrarectal (grupo $1, \mathrm{n}=71$ ) y el otro placebo, lubricante sin anestesia, (grupo $2, \mathrm{n}=28$ ), diez minutos antes del procedimiento.

RESULTADOS: La mediana global de dolor fue de 3,7 ( 0 no dolor y 10 máximo de dolor) y de molestias de 3,5. Los pacientes del grupo 1 (Xylocaína ${ }^{\circledR}$ ) mostraron una tendencia a presentar menos dolor y molestias aunque no alcanzó el grado de significación deseado ( $\mathrm{p}=0,7 \mathrm{y} \mathrm{p}=0,5$, respectivamente).

CONCLUSIONES: En nuestro medio no obtenemos los resultados descritos por otros grupos en cuanto a la disminución del dolor con el uso de gel intrarectal de anestesia tópica. No hemos encontrado diferencias significativas en cuanto a nivel de PSA, biopsias previas realizadas, uso del gel de lidocaína y grado de información recibida que afecten al grado de molestias y dolor.

PALABRAS CLAVE: Biopsias próstata. Dolor. Lidocaína intrarectal.

\section{ABSTRACT}

THE USE OF TRANSRECTAL LIDOCAINE IN TRANSRECTAL BIOPSIES OF THE PROSTATE

OBJECTIVE: To know in a quantitative manner the degree of discomfort and pain of the biopsies of the prostate and to evaluate the effectiveness of the transrectal lidocaine.

MATERIAL AND METHOD: We performed 140 transrectal biopsies of the prostate. Patients were included on a random basis into two arms: one of them received intrarectal lidocaine, $20 \mathrm{mg}$ (group $1, \mathrm{n}=71$ ) and the other group received placebo (group $2, \mathrm{n}=28$ ) both of them ten minutes prior the proceeding.

RESULTS: The global pain mean was 3.7 (0 no pain, 10 highest pain) and the global discomfort mean was 3.5. The group 1 patients showed a trend to feel less pain and discomfort although it did not reach the necessary statistic significance ( $\mathrm{p}=0,7 \mathrm{y} p=0,5$ respectively).

CONCLUSIONS: We do not achieve the good results obtained by other groups in order to decrease the degree of pain and discomfort with the use of intrarectal lidocaine. We did not find relationship between the PSA level, previous biopsies, intrarectal lidocaina and degree of information received and the degree of pain and discomfort.

KEY WORDS: Prostate biopsies. Pain. Intrarectal lidocaine. 
$\mathrm{E}_{\mathrm{t}}^{1}$ cáncer de próstata representa el cuarto tumor maligno más frecuente en el hombre y la segunda causa de muerte por cáncer en varones en Estados Unidos. Se calcula una incidencia de 110 nuevos casos por cada 100.000 habitantes en ese país, con una mortalidad en España de $13 / 100.000$ habitantes ${ }^{5,8}$. El procedimiento globalmente aceptado para su diagnóstico son las biopsias ecodirigidas transrectales de próstata. Se estima que unas 500.000 biopsias de próstata fueron realizadas en los Estados Unidos el pasado año ${ }^{5}$.

La mayor parte (65-90\%) de los pacientes sometidos a biopsias transrectales de próstata refieren algún tipo de molestias ${ }^{4,6}$, y aunque, en general, es un proceso bastante bien tolerado por los pacientes, un porcentaje de ellos que varía del 3 al 20\% según autores ${ }^{3,4}$ prefieren que se realice algún tipo de técnica analgésica asociada debido a un alto grado de molestias y dolor. Se han propuesto diferentes técnicas analgésicas para disminuir el grado de dolor y molestias entre las que se incluyen la inyección transrectal de lidocaína y la aplicación transrectal de gel de lidocaina ${ }^{1,7}$.

Con este estudio valoramos de una manera cuantitativa el grado de molestias y dolor de este procedimiento en nuestras manos y evaluamos la eficacia del gel de lidocaína intrarectal que con tan buenos resultados han obtenido otros gru$\operatorname{pos}^{1}$.

\section{MATERIAL Y MÉTODOS}

Realizamos un total de 140 biopsias transrectales de próstata ecodirigidas (BTPE) en ocho días consecutivos por sospecha de adenocarcinoma prostático. A los pacientes se les administra una preparación consistente en un antibiótico de amplio espectro la noche antes y la mañana del procedimiento y un enema de limpieza la mañana de la biopsia. Todas las intervenciones fueron realizadas por médicos internos residentes del servicio, en régimen ambulante y en posición de litotomía forzada. El ecógrafo utilizado fue un Siemens ${ }^{\circledR}$ sonoline SI200. Mediante una sonda transrectal biplanar de frecuencia variable de 5 a 7,5 MHz tomamos medidas de la próstata y realizamos biopsias sextantes y de áreas sospechosas ecodirigidas.

Los pacientes fueron incluidos de forma aleatoria y homogénea en dos brazos, uno recibió gel de lidocaína, $20 \mathrm{mg}$ (Xylocaína ${ }^{\circledR}$ ), intrarectal (grupo $1, \mathrm{n}=71$ ) y el otro placebo, lubricante sin anestesia, (grupo 2, n = 28), diez minutos antes del procedimiento. No se utilizó ningún tipo de analgesia o sedación durante la intervención.

Después de las biopsias se evaluó el grado de dolor, molestias e información recibida previamente mediante una encuesta visual lineal analógica $^{2}$ (Fig. 1).

\section{RESULTADOS}

La edad media de los varones fue de 68 años (43-82 años) con una mediana del antígeno específico prostático (P.S.A.) de 10,6 (1,5-181 $\mathrm{ng} / \mathrm{ml})$. El volumen medio de la glándula fue de 52,9 cc (13-167). La mediana global de dolor fue en la escala analógica lineal de 3,7 ( 0 no dolor y 10 máximo de dolor) y de molestias de 3,5 (Figs. 2 y 3). En el 30\% de los casos (43/140) fue su segunda biopsia. Los pacientes del grupo 1 (Xylocaína ${ }^{\circledR}$ ) mostraron una tendencia a presentar menos dolor y molestias aunque no alcanzó

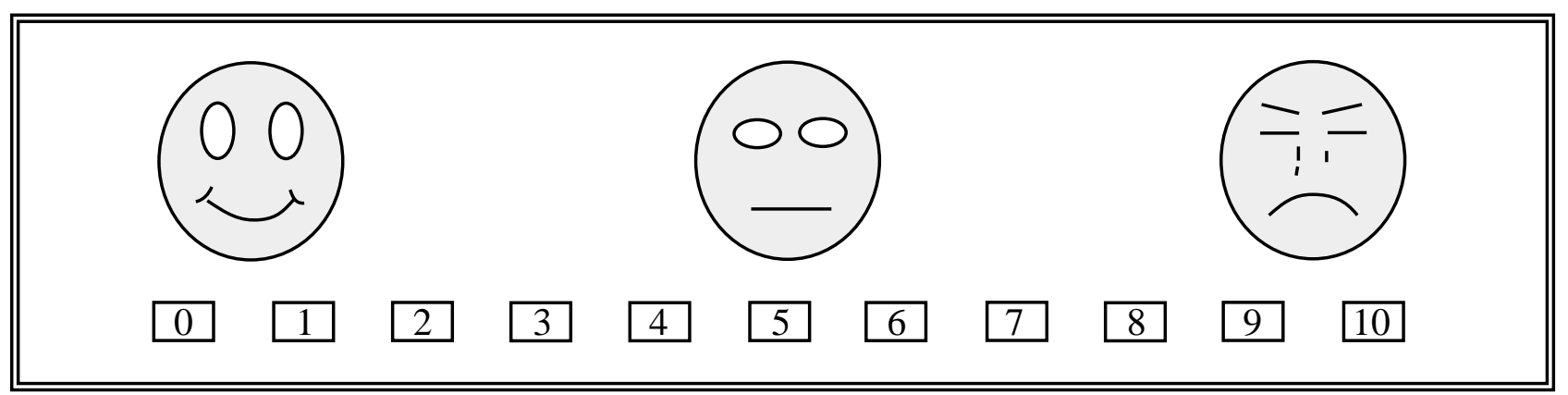

Escala analógica Linea Visual usada para valorar el grado de dolor y de molestias ${ }^{1}$. 


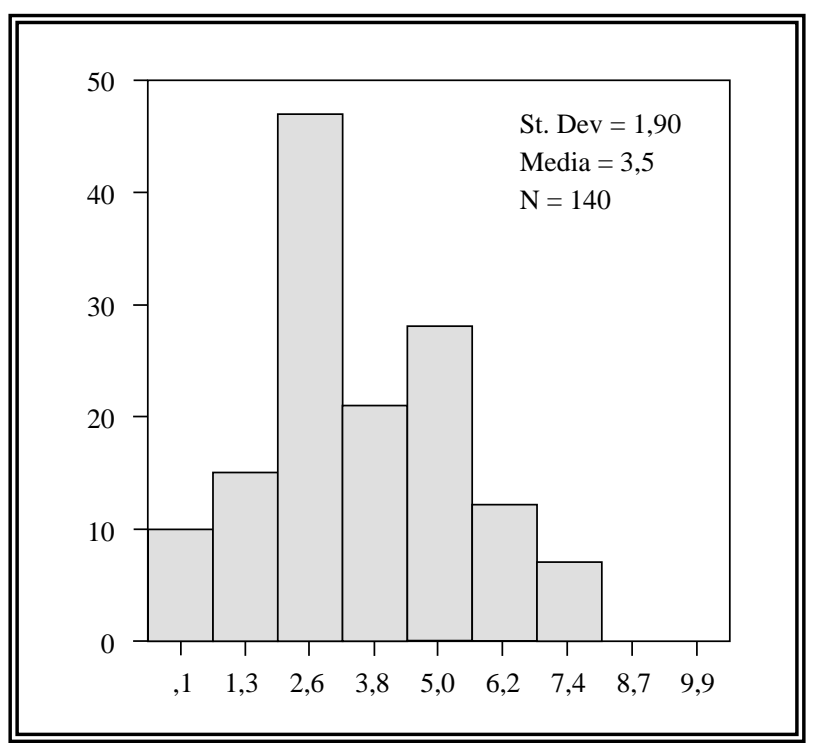

FIGURA 2. Histograma mostrando el grado de dolor global de las biopsias transrectales de próstata ecodirigidas.

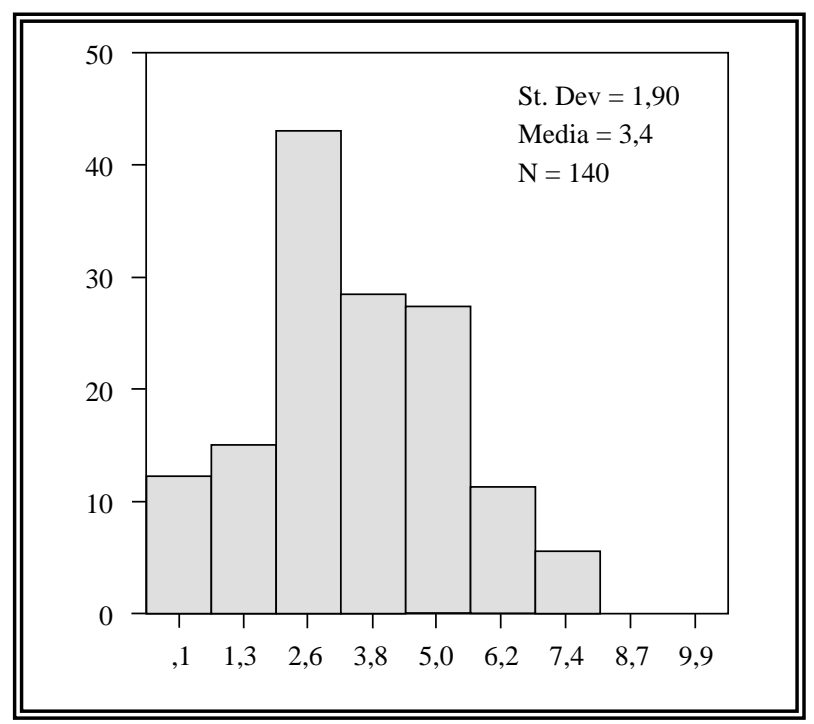

FIGURA 3. Histograma mostrando el grado de molestias global de las biopsias transrectales de próstata ecodirigidas.

el grado de significación deseado $(p=0,7$ y p $=$ 0,5 respectivamente) (Fig. 4). Los pacientes demostraron estar muy bien informados del procedimiento a realizar, grado medio de información de 8,01 (0-10). Las herramientas estadísticas utilizadas fueron la $\mathrm{t}$ de Student para comparación de medias y ANOVA y Chi 2 para valorar el efecto de la información recibida y de las biopsias previas sobre el grado de dolor y molestias (SPSS 8.0).

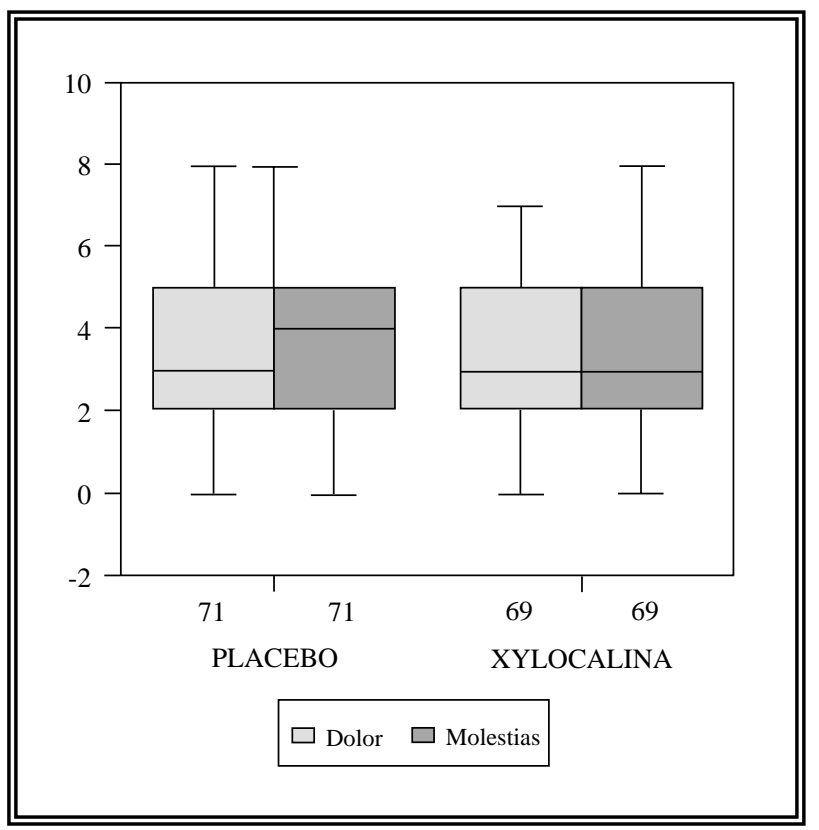

FIGURA 4. Diagrama de cajas en donde se aprecia una tendencia a reducir el grado de molestias en el grupo tratado con gel anestésico aunque no alcanza el grado de significación necesaria.

\section{DISCUSIÓN}

En nuestro medio no obtenemos los resultados descritos por otros grupos en cuanto a la disminución del dolor con la administración intrarectal de gel de lidocaína ${ }^{1}$, aunque hemos observado una tendencia (no significativa) a la disminución de molestias en el grupo 1. Casi un $20 \%$ de los procedimientos fueron realizados por residentes de $2^{\circ}$ año al poco de llegar al Servicio, con menos experiencia que los residentes de $4^{\circ}$ y $5^{\circ}$ año, lo cual podría explicar el leve incremento de dolor comparado con otras series $^{1}$.

Aunque no hemos podido demostrar mediante regresión logística una relación estadísticamente significativa entre grado de información y puntuación de dolor y molestias en la escala lineal (p $=0,776$ ) consideramos que es importante una correcta información del procedimiento a realizar, ya que disminuye mucho la ansiedad del paciente ${ }^{4}$. Nuestros pacientes demostraron estar muy bien informados previamente y además se les fue informando in situ antes de realizar cada paso (tacto rectal, introducción del transductor, ecografía con toma de medidas prostáticas y biopsias). Toda esta información recibida les 
tranquiliza en gran manera, lo cual disminuye su ansiedad y aumenta la tolerabilidad del paciente al procedimiento.

En nuestro estudio no excluimos a los pacientes a los que se les había realizado previamente biopsias. Issa y cols. ${ }^{1}$ excluyeron a este subgrupo de pacientes al inferir que la absorción del gel anestésico por la mucosa rectal hasta los nervios capsulares prostáticos podría estar disminuida por fibrosis de las biopsias previamente realizadas. En un análisis multivariante nosotros no hemos encontrado diferencias significativas entre los pacientes con y sin biopsias previas en cuanto al grado de dolor y molestias al utilizar el gel de lidocaína ( $\mathrm{p}=0,677)$.

Debido a la gran área poblacional que atiende nuestro centro nos encontramos una gran variabilidad en los niveles socioculturales por lo que ha podido influir a la hora de rellenar la encuesta (cuatro pacientes no sabían leer y tuvieron que ser ayudados por nuestro personal de enfermería) aunque no debería suponer un sesgo debido a la homogenización de la muestra. Ambos brazos fueron equivalentes tanto en biopsias previas realizadas, como en nivel de PSA y en tacto rectal (sospechoso / no sospechoso) controlando así los posibles factores de confusión de la muestra.

En un medio con mucha presión asistencial, en donde no hay que perder de vista las listas de espera y la ocupación de quirófano hay que buscar un equilibrio entre tolerabilidad del procedimiento (confort y seguridad para el paciente) y la rentabilidad del mismo (ocupación de quirófanogestión de la lista de espera). En una jornada laboral realizamos entre 16 y 20 biopsias transrectales de próstata ecodirigidas asumiendo un grado leve-moderado de dolor y molestias sin usar anestesia tópica. El uso de ésta, al tener que esperar los diez minutos necesarios para que haga efecto supondría realizar entre 8 y 9 procedimientos menos, disminuyendo el rendimiento de quirófano en un $50 \%$.

\section{CONCLUSIONES}

En nuestro medio no obtenemos los resultados descritos por otros grupos en cuanto a la disminución del dolor con el uso de gel intrarectal de anestesia tópica. No hemos encontrado diferencias significativas en cuanto a nivel de PSA, biopsias previas realizadas, uso o no de gel de lidocaína y grado de información recibida que afecten al grado de molestias y dolor del procedimiento. Sin embargo, el grado de dolor y molestia fueron tan leves en nuestra experiencia que no creemos rentable la administración previa intrarectal de anestesia tópica en forma de gel. De esta manera, asumiendo el grado leve de molestias se pueden realizar el doble de procedimientos en una jornada mejorando el rendimiento de quirófano.

Agradecimientos: Este trabajo no podría haberse realizado sin la colaboración de las Srtas. A. Barrio y C. López, D.U.E.s del Servicio de Urología del Hospital La Paz.

\section{REFERENCIAS}

1. MUTA M. ISSA, SAJIT BUX, CHUN T et al.: A randomized prospective trial of intrarectal lidocaine for pain control during transrectal prostate biopsy: the emory university eperience. J Urol 2000; 164: 397.

2. REVILL SI, ROBINSON JO, ROSEN M et al.: The reliability of a linear analogue for evaluating pain. Anesthesia 1976; 31: 1191.

3. IRANI J, FOURNIER F, BON D et al.: Patient tolerance of transrectal ultrasound-guided biopsy of the prostate. Br J Urol 1997; 79: 608.

4. COLLINS GN, LLOYD SN, HEHIR M et al.: Multiple transrectal ultrasound-guided prostatic biopsies. True morbidity and patient acceptance. $\mathrm{Br} J$ Urol 1993; 71: 460.

5. NAITOH J, ZEINER RL, DEKERNION JB.: Diagnosis and treatment of prostate cancer. Am Fam Phys 1998; 57: 1531.

6. CLEMENTS R, AIDEYAN OU, GRIFFITHS GJ, PEELING WB.: Side effects and patient acceptability of transrectal biopsy of the prostate. Clin Rad 1993; 47: 125.

7. NASH P, BRUCE J, INDUDHARA R, SHINOHARA K.: Transrectal ultrasound guided prostatic nerve blockade eases systematic needle biopsy of the prostate. J Urol 1996; 155: 607.

8. WALSH RETIK, VAUGHAN WEIN.: Campbell's Urology. $8^{\text {th }}$ Edition (CD). Saunders 2003; 14 (11), Chapter 85.

Dr. J.M. García Mediero

C/ Jorge Juan, 84 - 6C

28009 Madrid

(Trabajo recibido el 29 julio de 2003) 